\title{
Irradiation history and resulting isotope decay scheme influence on yttrium gamma activity
}

\author{
S. Kilim¹, M. Bielewicz, A. Polanski, E. Strugalska-Gola, M. Szuta, A. \\ Wojciechowski \\ National Centre for Nuclear Research \\ Otwock-Świerk 05-400, Poland \\ E-mail: s.kilim@cyf.gov.pl; m.bielewicz@cyf.gov.pl; polanski@jinr.ru; \\ elasg@cyf.gov.pl; mszuta@cyf.gov.pl; andrzej@cyf.gov.pl
}

\section{J. Adam, M. Kadykov, V. Pronskich, S. Tyutyunnikov, W. Furman}

Joint Institute of Nuclear Research

141980 Dubna, Russia

E-mail: jadamenusun.jinr.ru; kadykovejinr.ruł; vspronefnal.gov;

tsiesunse.jinr.ru; furmanedubna.ru

\section{Wagner, O. Svoboda}

Nuclear Physics Institute of CAS

25068 Rez, Czech Republic

E-mail: wagner@ujf.cas.cz; svobodalujf.cas.cz

\section{Chilap}

CPTP “Atomenergomash”

Moscow, Russia

E-mail: chilap@cftp-aem.ru

Spallation neutrons produced during QUINTA setup irradiation react with ${ }^{89} \mathrm{Y}$ samples producing a set of gamma active yttrium isotopes. These reactions are used to determine neutron fields inside setup. The measured gamma activity used for isotope production determination is influenced by several phenomena. This work is focused on two of them - on irradiation history (IH) and decay scheme. After introductory explanation of the both effects some QUINTA experiment results are shown as the illustrative examples.

XXI International Baldin Seminar on High Energy Physics Problems

JINR, Dubna, Russia

September 10-15, 20

Speaker 


\section{Introduction}

The activation detectors based on yttrium are used together with others for neutron production studies during QUINTA setup experiments. The basic reactions taken into account at yttrium sample activation are of type ${ }^{89} \mathrm{Y}(\mathrm{n}, \mathrm{Xn})$. The resulting isotopes $\operatorname{are}{ }^{88} \mathrm{Y},{ }^{87} \mathrm{Y},{ }^{86} \mathrm{Y}$ and ${ }^{85} \mathrm{Y}$ and $\mathrm{x}=2, \ldots, 5$ respectively. The reaction yield or in other words determination of production rate of resulting isotope is based on sample gamma spectra measurements. Each isotope can be identified by the energy of emitted gamma quanta i.e. by energy of the peak in the gamma spectrum.

Table $1 .{ }^{89} \mathrm{Y}(\mathrm{n}, \mathrm{xn})$ reaction basic data.

\begin{tabular}{|c|c|c|c|c|c|}
\hline Reaction & $\begin{array}{c}\text { Produced } \\
\text { Isotope }\end{array}$ & T1/2 & $\begin{array}{c}\text { Reaction } \\
\text { Threshold } \\
{[\mathrm{MeV}]}\end{array}$ & $\begin{array}{c}\gamma \text {-line } \\
\text { Energy } \\
{[\mathrm{keV}]}\end{array}$ & $\begin{array}{c}\gamma \text {-line } \\
\text { Intensity } \\
{[\%]}\end{array}$ \\
\hline \multirow{2}{*}{${ }^{89} Y(n, 2 n)$} & \multirow{2}{*}{${ }^{88} \mathrm{Y}$} & \multirow{2}{*}{$106.65 d$} & \multirow{2}{*}{11.5} & 898.042 & 93.7 \\
\hline & & & & 1836.063 & 99.2 \\
\hline \multirow{3}{*}{${ }^{89} Y(n, 3 n)$} & ${ }^{87} \mathrm{mY}$ & $13.37 \mathrm{~h}$ & 20.8 & 380.79 & 78 \\
\hline & \multirow{2}{*}{${ }^{87} \mathrm{Y}$} & \multirow{2}{*}{$79.8 \mathrm{~h}$} & \multirow{2}{*}{20.8} & 388.53 & 82.00 \\
\hline & & & & 484.805 & 89.7 \\
\hline${ }^{89} Y(n, 4 n)$ & ${ }^{86} \mathrm{Y}$ & $14.74 \mathrm{~h}$ & 32.7 & 1076.64 & 82.00 \\
\hline \multirow{2}{*}{${ }^{89} Y(n, 5 n)$} & \multirow{2}{*}{${ }^{85} \mathrm{Y}$} & $2.68 \mathrm{~h}$ & 42.6 & 231.67 & 84.00 \\
\hline & & $4.86 \mathrm{~h}$ & 42.6 & 231.67 & 22.8 \\
\hline
\end{tabular}

\section{Irradiation history effect on sample activity}

To make the isotope quantities comparable between themselves the measured values have to be corrected for several effects. These corrections are described in many gamma spectrometry textbooks and manuals (for example nice introduction of Gordon Gilmore [1]). We use standardly needed spectrometry correction during all experiments. More specifically our way of correction description is shown at [2,3]. Here we concentrate on two specific corrections important in our case: correction for beam instability during irradiation and production of isomeric states.

Assigning symbol $I_{k}$ to isotope $k$ production rate per gram of sample one has:

$$
I_{k}=\frac{N_{A} \sigma_{k}}{A}
$$

where $N_{A}$ - Avogadro's number, number of nuclei in one gram-atom;

$A$ - parent isotope atomic number, gram-atom, [g];

$\sigma_{k}-$ reaction cross section, $\left[\mathrm{cm}^{2}\right]$;

$I_{k}-$ isotope $k$ production rate, $\left[\mathrm{cm}^{2} / \mathrm{g}\right]$. 
In our case the $I_{k}$ is the final parameter looked for. Implicitly this rate is per one beam deuteron. For discussion purpose we'll use an intermediate parameter $N_{k}\left(t_{i r}\right)$, the resulting isotope $k$ number of nuclei at the end of irradiation.

Deuteron beam produced by NUCLOTRON is a sequence of pulses of intensity about $10^{8}$ $10^{10}$ deuterons and frequency $0.125 \mathrm{~Hz}$. The NUCLOTRON staff gives us the pulse intensity $\varphi_{i}$ and time of the pulse appearance $-t_{i}$. The $i$-th pulse produces $\frac{N_{A} \sigma_{k}}{A} \varphi_{i}=I_{k} \varphi_{i}$ nuclei of isotope $k$ in a gram of sample material. As the isotope $k$ decays, the $i$-th pulse contribution to the isotope $k$ quantity at the end of irradiation is only $I_{k} \varphi_{i} e^{-\lambda_{k}\left(t_{i r}-t_{i}\right)}$. The total isotope $k$ quantity at the end of irradiation amounts for sample with mass $m$ :

$$
N_{k}\left(t_{i r}\right)=m I_{k} \sum_{i=1}^{i r} \varphi_{i} e^{-\lambda_{k}\left(t_{i r}-t_{i}\right)}
$$

where $\lambda_{k}$ is the isotope $k$ decay constant, index $i$ numbers the beam pulses ( $i r$ is index number of last pulse) and $t_{i r}-t_{i}$ is the time from $i$-th pulse appearance till the end of irradiation. This formula will be referred to as a variable beam formula. In case of constant beam and regular pulses the number of particles in the $i$-th pulse can be expressed as $\varphi=\phi \frac{\Delta t}{t_{i r}}$ with $\phi$ being the beam integral, and $\Delta t$ the time from the beginning of one pulse till beginning of the next pulse. For $t_{i r}>>\Delta t$ the (2.2) formula becomes:

$$
N_{k}\left(t_{i r}\right)=m I_{k} \frac{\phi}{t_{i r}} \int_{0}^{t_{i j}} e^{-\lambda_{k}\left(t_{i r}-t\right)} d t=m I_{k} \phi \frac{1-e^{-\lambda_{k} t_{i r}}}{\lambda_{k} t_{i r}}
$$

The best way to illustrate the constant beam formula applicability or rather irradiation history influence is to create variable beam to constant beam formulae ratio (IHR).

$$
I H R=\frac{N_{k}\left(t_{i r}\right)_{V B}}{N_{k}\left(t_{i r}\right)_{C B}}=\frac{\lambda_{k} t_{i r}}{\phi\left(1-e^{-\lambda_{k} t_{i r}}\right.} \sum_{i=1}^{i r} \varphi_{i} e^{-\lambda_{k}\left(t_{i r}-t_{i}\right)}
$$

The indexes $V B$ and $C B$ refer to variable beam and constant beam respectively. 

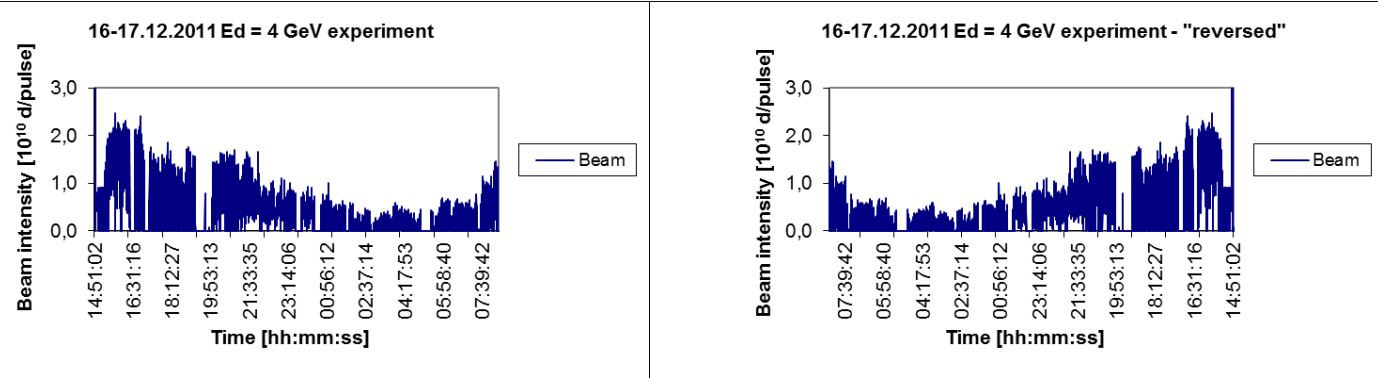

Fig. 1. QUINTA setup 16-17.12.2011 experiment beam run (left) and "reversed" beam run (right). Reversed means the first pulse on left graph becomes the last one on the right.

For illustration of irradiation history effect one experiment was selected - QUINTA setup experiment 16-17.12.2011. To make the effect more spectacular a mirror run was created named "reversed" - and compared with the real one (see Fig.1). In both cases the deuteron beam integral was $3.37 \times 10^{13}$ and irradiation time 17:31:00 [hh:mm:ss].

Table 2. 16-17.12.2011 experiment parameter IHR as the measure of irradiation history influence on yttrium isotope production

\begin{tabular}{|l|c|c|c|c|c|}
\hline Beam runlisotope & ${ }^{88} \mathrm{Y}$ & ${ }^{87 \mathrm{~m}} \mathrm{Y}$ & ${ }^{87} \mathrm{Y}$ & ${ }^{86} \mathrm{Y}$ & ${ }^{85} \mathrm{Y}$ \\
\hline $\begin{array}{l}\text { Beam run averaged } \\
\text { (constant) }\end{array}$ & 1.00 & 1.00 & 1.00 & 1.00 & 1.00 \\
\hline Real beam run & 1.00 & 0.84 & 0.97 & 0.86 & 0.55 \\
\hline Reversed beam run & 1.00 & 1.18 & 1.03 & 1.16 & 1.83 \\
\hline
\end{tabular}

The above displayed example shows very strong dependence on irradiation history for short-lived isotopes and rather weak for long-lived ones compared to irradiation time. Therefore it is very important to assess the average uncertainty on $N_{k}\left(t_{i r}\right)$ caused by irradiation history. The IH contribution to isotope production uncertainty does not depend on $\varphi_{i}$ uncertainty $\left(\Delta \varphi_{i}\right)$ nor on $t_{i}$ uncertainty $\left(\Delta t_{i}\right)$. More sensible assessment is like:

$$
\Delta N_{k}\left(t_{i r}\right)_{I H}=\left|\frac{N_{k}\left(t_{i r}\right)_{V B}-N_{k}\left(t_{i r}\right)_{C B}}{N_{k}\left(t_{i r}\right)_{C B}}\right|=|I H R-1|
$$

\section{Influence of the isotope decay scheme on measured production}

Despite of ${ }^{87 \mathrm{~m}} \mathrm{Y}$ being an isomeric state of the same nucleus ${ }^{87} \mathrm{Y}$ their de-excitation and decay process can be described as if they are different isotopes. Isotope ${ }^{87 \mathrm{~m}} \mathrm{Y}$ will be indexed as "1" and the ${ }^{87} \mathrm{Y}$ one as " 2 ". Both isotopes are produced in ${ }^{89} \mathrm{Y}(\mathrm{n}, 3 \mathrm{n})$ reaction and then both of them decay as in Fig. 2. The ${ }^{87 m} \mathrm{Y}$ transits first into ${ }^{87} \mathrm{Y}$ and then decays. 


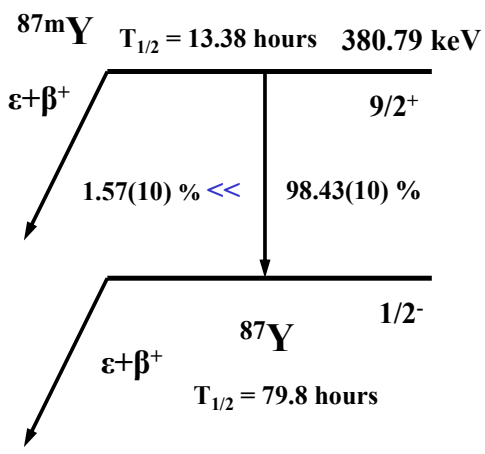

Fig. 2. The ${ }^{87 \mathrm{~m}} \mathrm{Y}$ isomeric transition and decay scheme. The isomer state gamma transition to ground state is dominant in this case.

Each sample activity is measured two times, usually. The first measurement is done as soon as possible after the irradiation to "catch" the short-lived isotopes and then follows the second one, much longer, to measure the long-lived. In case of ${ }^{87} \mathrm{Y}$ the first measurement is performed while the ${ }^{87 \mathrm{~m}} \mathrm{Y}$ decays yet. Neglecting the ${ }^{87 \mathrm{~m}} \mathrm{Y}$ effect leads to discrepancy between series 1 and series 2 results of ${ }^{87} \mathrm{Y}$.

Assuming constant deuteron beam and constant neutron flux $\varphi$ one can express the mentioned isotopes quantity dependence on time with two sets of equations.

$$
\begin{aligned}
& \frac{d N_{1}}{d t}=m I_{1} \varphi-\lambda_{1} N_{1} \\
& \frac{d N_{2 \text { from } 1}}{d t}=\lambda_{1} N_{1}-\lambda_{2} N_{2 \text { from } 1} \mathrm{t} \leq \mathrm{t}_{\text {irr }} \\
& \frac{d N_{2 \text { direct }}}{d t}=m I_{2} \varphi-\lambda_{2} N_{2 \text { direct }} \\
& \frac{d N_{1}}{d t}=-\lambda_{1} N_{1} \\
& \frac{d N_{2 \text { from } 1}}{d t}=\lambda_{1} N_{1}-\lambda_{2} N_{2 \text { from } 1} \mathrm{t}>\mathrm{t}_{\text {irr }} \\
& \frac{d N_{2 \text { direct }}}{d t}=-\lambda_{2} N_{2 \text { direct }} \\
& N_{2}=N_{2 \text { from } 1}+N_{2 \text { direct }}
\end{aligned}
$$

Here $\lambda_{1}$ and $\lambda_{2}$ are the decay constants, $N_{2 \text { direct }}$ - nuclei quantity produced directly from (n,3n) reaction, $N_{2 \text { from } 1}$ - produced from $N_{1}$ decay. The first set refers to irradiation period and the second to post-irradiation decay. Term $m I_{1,2} \varphi$ describes direct production rate of isotope 1,2 while $\lambda_{1} N_{1}$ describes both isotope 1 decay rate and isotope 2 production rate from isotope 1 decay. 
Solving the above equation sets one gets for irradiation time $t_{i r}$ :

$$
\begin{aligned}
& N_{1}\left(t_{i r}\right)=m I_{1} \phi \frac{\left(1-e^{-\lambda_{1} t_{i r}}\right)}{\lambda_{1} t_{i r}} \\
& N_{2 \text { froml }}\left(t_{i r}\right)=m I_{1} \phi\left[\frac{1-e^{-\lambda_{2} t_{i r}}}{\lambda_{2} t_{i r}}-\frac{e^{-\lambda_{2} t_{i r}}-e^{-\lambda_{1} t_{i r}}}{\left(\lambda_{1}-\lambda_{2}\right) t_{i r}}\right] \\
& N_{2 \text { direct }}\left(t_{i r}\right)=m I_{2} \phi \frac{\left(1-e^{-\lambda_{2} t_{i r}}\right)}{\lambda_{2} t_{i r}} \\
& N_{2}\left(t_{\text {ir }}\right)=N_{2 \text { froml }}\left(t_{\text {ir }}\right)+N_{2 \text { direct }}\left(t_{i r}\right)
\end{aligned}
$$

For the time after irradiation, $t_{+}$the formulae look like:

$$
\begin{aligned}
& N_{1}\left(t_{+}\right)=N_{1}\left(t_{i r}\right) e^{-\lambda_{1} t_{+}} \\
& N_{2 \text { from } 1}\left(t_{+}\right)=N_{1}\left(t_{i r}\right) \frac{\lambda_{1}}{\left(\lambda_{1}-\lambda_{2}\right)}\left(e^{-\lambda_{2} t_{+}}-e^{-\lambda_{1} t_{+}}\right) \\
& N_{2}\left(t_{+}\right)=N_{2}\left(t_{i r}\right) e^{-\lambda_{2} t_{+}}+N_{1}\left(t_{i r}\right) \frac{\lambda_{1}}{\left(\lambda_{1}-\lambda_{2}\right)}\left(e^{-\lambda_{2} t_{+}}-e^{-\lambda_{1} t_{+}}\right)
\end{aligned}
$$

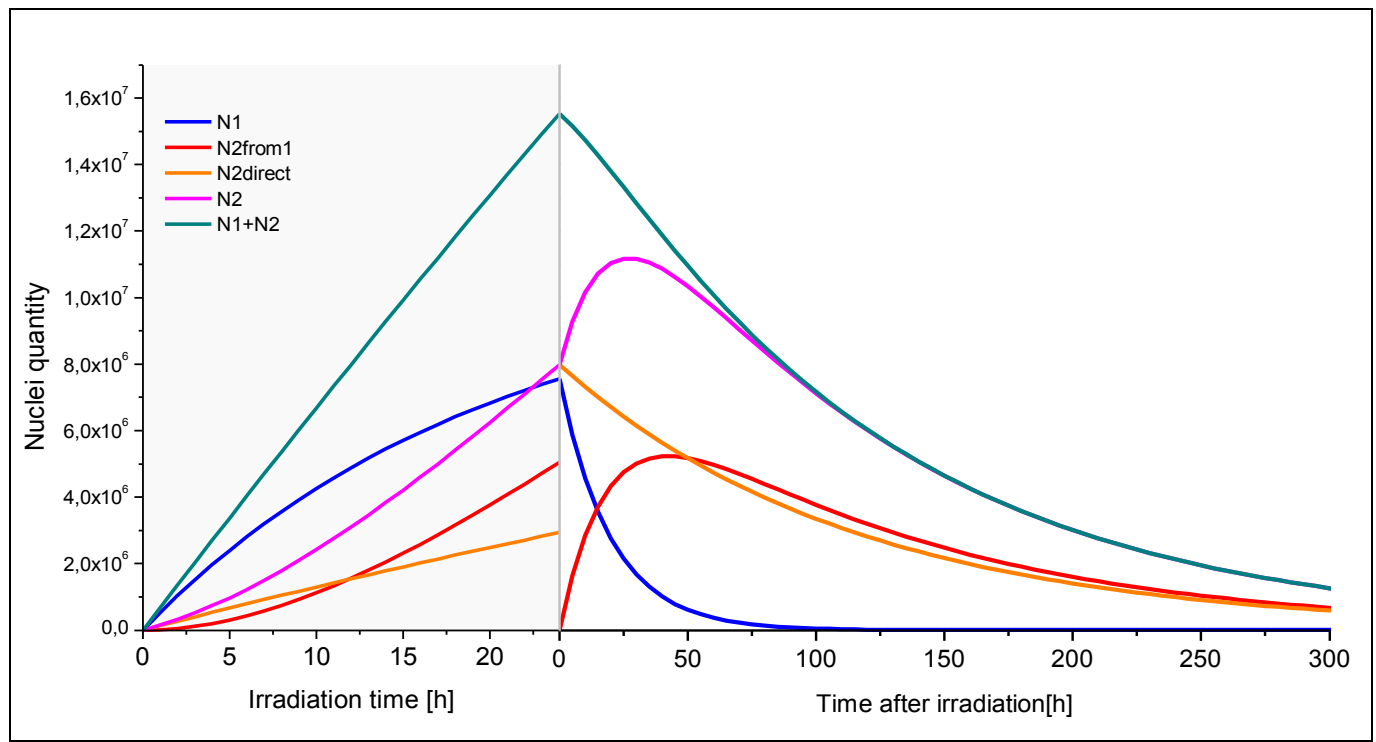

Fig. 3. Time dependence of ${ }^{87 \mathrm{~m}} \mathrm{Y}$ and ${ }^{87} \mathrm{Y}$ quantity during ${ }^{89} \mathrm{Y}$ sample irradiation and after end of irradiation.

As ${ }^{87 \mathrm{~m}} \mathrm{Y}$ and ${ }^{87} \mathrm{Y}$ are the two different states of the same isotope ${ }^{87} \mathrm{Y}$ it makes sense to use function $N_{2 \text { total }}=N_{1}+N_{2}$ and $I_{2 \text { total }}=I_{1}+I_{2}$ to describe isotope quantity and production rate.

On the other side the mentioned above quantities of isotope nuclei are related to respective peak area $-S$ corrected for gamma line intensity $-I_{p}$ gamma spectrometer efficiency $-\varepsilon_{p}$, 
coefficient of coincidence $-C O I$, real time of measurement $t_{\text {real }}$ and the live one $-t_{\text {live, }}$ by formula:

$$
N_{k}\left(t_{+}\right)=S \frac{100}{I_{\gamma} \varepsilon_{p} \operatorname{COI}\left(1-e^{-\lambda_{k} t_{\text {real }}}\right)} \frac{t_{\text {real }}}{t_{\text {live }}}
$$

After recalculation the isotope quantities to isotope production rate one gets:

$$
\begin{aligned}
& I_{1}=\frac{N_{1}\left(t_{+}\right)}{m \phi} \frac{\lambda_{1} t_{i r}}{\left(1-e^{-\lambda_{1} t_{i r}}\right)} e^{\lambda_{1} t_{+}} \\
& I_{2 \text { total }}=\frac{N_{2}\left(t_{+}\right)}{m \phi} \frac{\lambda_{2} t_{i r}}{\left(1-e^{-\lambda_{2} t_{i r}}\right)} e^{\lambda_{2} t_{+}}+I_{1} \frac{\lambda_{2}}{\left(\lambda_{1}-\lambda_{2}\right)}\left[\frac{\left(1-e^{-\lambda_{1} t_{i r}}\right.}{\left(1-e^{-\lambda_{2} t_{i r}}\right)} e^{-\left(\lambda_{1}-\lambda_{2}\right) t_{+}}-1\right]
\end{aligned}
$$

In case of variable beam run the production process description should be modified. Each beam pulse contribution to $N_{k}\left(t_{i r}\right)$ should be treated separately. For the $i$-th pulse, $\varphi_{i}$ contributes $m I_{1} \varphi_{i} e^{-\lambda_{1}\left(t_{i r}-t_{i}\right)}$ to $N_{1}\left(t_{i r}\right)$ and $m I_{1} \frac{\lambda_{1}}{\left(\lambda_{1}-\lambda_{2}\right)}\left(e^{-\lambda_{2}\left(t_{i r}-t_{i}\right)}-e^{-\lambda_{1}\left(t_{i r}-t_{i}\right)}\right)+m I_{2} \varphi_{i} e^{-\lambda_{2}\left(t_{i r}-t_{i}\right)}$ to $N_{2}\left(t_{\text {ir }}\right)$

$$
\begin{aligned}
& N_{1}\left(t_{i r}\right)=m I_{1} \sum_{t_{i}=t_{1}}^{t_{i r}} \varphi_{i} e^{-\lambda_{k}\left(t_{i r}-t_{i}\right)} \\
& N_{2 \text { froml }}\left(t_{i r}\right)=m I_{1} \frac{\lambda_{1}}{\left(\lambda_{1}-\lambda_{2}\right)} \sum_{i=1}^{i r} \varphi_{i}\left(e^{-\lambda_{2}\left(t_{i r}-t_{i}\right)}-e^{-\lambda_{1}\left(t_{i r}-t_{i}\right)}\right) \\
& N_{2 \text { direct }}\left(t_{\text {ir }}\right)=m I_{2} \sum_{i=1}^{i r} \varphi_{i} e^{-\lambda_{2}\left(t_{i r}-t_{i}\right)} \\
& N_{2}\left(t_{\text {ir }}\right)=N_{2 \text { froml }}\left(t_{i r}\right)+N_{2 \text { direct }}\left(t_{i r}\right)
\end{aligned}
$$

The formulae describing post-irradiation decay process remain unchanged. Combining (3.5) with (3.9) one gets formulae for isotope production rates:

$$
\begin{aligned}
& I_{1}=\frac{N_{1}\left(t_{+}\right)}{m} \frac{e^{\lambda_{1} t_{+}}}{\sum_{i=1}^{i r} \varphi_{i} e^{-\lambda_{1}\left(t_{i r}-t_{i}\right)}} \\
& I_{2 \text { total }}=\frac{N_{2}\left(t_{+}\right) e^{\lambda_{2} t_{+}}}{m \sum_{i=1}^{i r} \varphi_{i} e^{-\lambda_{2}\left(t_{i r}-t_{l}\right)}}+\frac{I_{1}}{\left(\lambda_{1}-\lambda_{2}\right)}\left[\lambda_{1} \frac{\sum_{i=1}^{i r} \varphi_{i} e^{-\lambda_{1}\left(t_{i r}-t_{i}\right)}}{\sum_{i=1}^{i r} \varphi_{i} e^{-\lambda_{2}\left(t_{i r}-t_{l}\right)}} e^{-\left(\lambda_{1}-\lambda_{2}\right) t_{+}}-\lambda_{2}\right]
\end{aligned}
$$

The effect of correction for decay scheme is illustrated on example ${ }^{87} \mathrm{Y}$ production axial distribution got from one of QUINTA experiments - Fig. 4. 


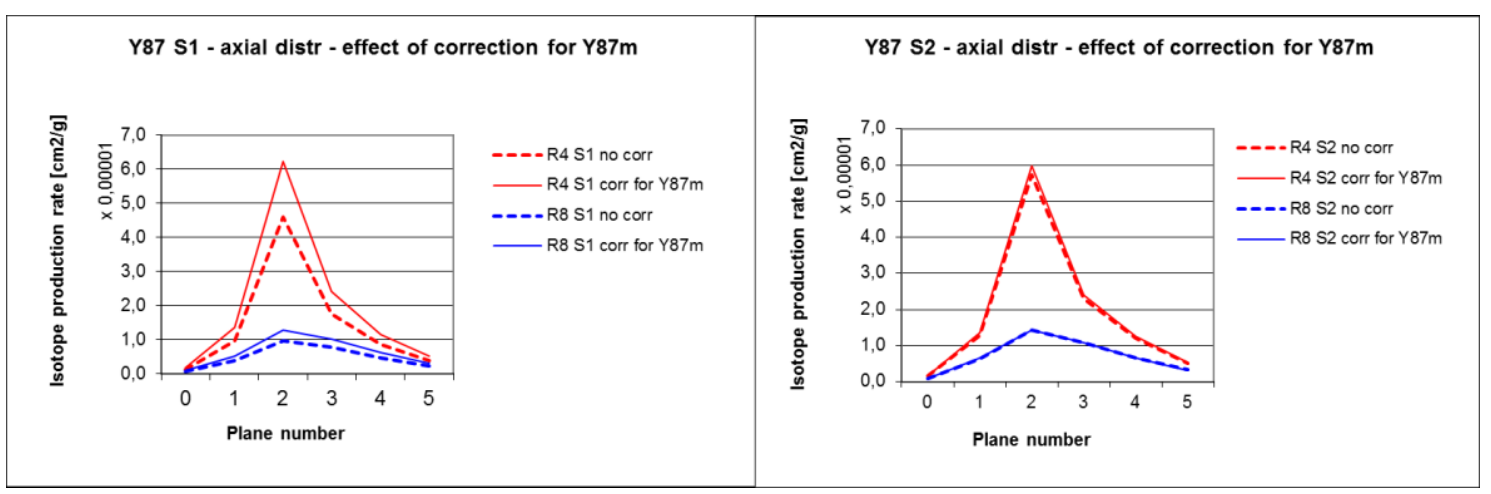

Fig. 4. Experiment QUINTA 2011-03-20-21 $6 \mathrm{GeV}-{ }^{87} \mathrm{Y}$ production series 1 and 2 measurement axial distributions as illustration of correction for decay ${ }^{87 \mathrm{~m}} \mathrm{Y} \rightarrow{ }^{87} \mathrm{Y}$ effect. The dashed lines stand for results not corrected results.

Measured gamma spectra were evaluated in the code DEIMOS [5] and consequent analysis was done in EXCEL using the equations mentioned above.

\section{Conclusions}

As expected the irradiation history effect on measured sample activity depends on resulting isotope half life time. Shorter the half time is larger is the effect of the irradiation history. As concerns ${ }^{89} \mathrm{Y}(\mathrm{n}, \mathrm{xn})$ reactions the irradiation history affects the most ${ }^{86} \mathrm{Y},{ }^{87 \mathrm{~m}} \mathrm{Y}$ and ${ }^{85} \mathrm{Y}$ production.

Influence of decay scheme effect of produced isotope on measured activity depends on time interval between the end of irradiation and start of measurement, on cooling time. Shorter the cooling time is larger is the decay scheme effect and the appropriate correction.

In case of QUINTA experiments where the irradiation times are of order 12-24 hours both effects are necessarily taken into account.

\section{References}

[1] Gordon Gilmore: Practical Gamma-ray Spectrometry, Wiley, 2008

[2] M. Majerle et al: Monte-Carlo method in Neutron Activation Analysis, E15-2009-178, JINR Dubna preprint (http://wwwinfo.jinr.ru/publish/Preprints/2009/178(E11-2009-178).pdf)

[3] O. Svoboda et al., Study of spallation reactions, neutron production and transport in thick lead target and uranium blanket irradiated with 0.7 GeV protons, E15-2009-177, JINR Dubna preprint (http://wwwinfo.jinr.ru/publish/Preprints/2009/177(E15-2009-177).pdf )

[4] V. Wagner te al: Measurements of Neutrons in Different Pb/U Setups Irradiated by Relativistic Protons and Deuterons by means of Activation Samples, Journal of Physics: Conference series 366 (2012) 012047, DOI: 10.1088/1742-6596/366/1/012047

[5] J. Frána, Program DEIMOS32 for Gamma-Ray Spectra Evaluation, J. Rad. Nucl. Chem., V. 257, No. 3 P. (2003) 583-587. 